\title{
The Coupled Effect of Loading Rate and Grain Size on Tensile Strength of Sandstones under Dynamic Disturbance
}

\author{
Miao Yu, Chenhui Wei, and Leilei Niu \\ School of Resources and Civil Engineering, Northeastern University, Shenyang 110819, China \\ Correspondence should be addressed to Miao Yu; 741975392@qq.com
}

Received 26 November 2016; Revised 17 February 2017; Accepted 20 February 2017; Published 15 March 2017

Academic Editor: Yuri S. Karinski

Copyright (C) 2017 Miao Yu et al. This is an open access article distributed under the Creative Commons Attribution License, which permits unrestricted use, distribution, and reproduction in any medium, provided the original work is properly cited.

\begin{abstract}
It is of significance to comprehend the effects of rock microstructure on the tensile strength under different loading rates caused by mining disturbance. So, in this paper, three kinds of sandstones drilled from surrounding rocks in Xiao Jihan Coal to simulate the in situ stress state, whose average grain size is $30 \mu \mathrm{m}$ (fine grain, FG), $105 \mu \mathrm{m}$ (medium grain, MG), and $231 \mu \mathrm{m}$ (Coarse grain, CG), are selected with the calculation of optical microscopic technique and moreover processed to Brazilian disc (BD) to study the mechanical response of samples. The dynamic Brazilian tests of samples with three kinds of grain sizes are conducted with the Split Hopkinson Pressure Bar (SHPB) driven by pendulum hammer, which can produce four different velocities $(V=2.0 \mathrm{~m} / \mathrm{s}, 2.5 \mathrm{~m} / \mathrm{s}$, $3.3 \mathrm{~m} / \mathrm{s}$, and $4.2 \mathrm{~m} / \mathrm{s}$ ) when the incident bar is impacted by pendulum hammer. The incident wave produced by pendulum hammer is a slowly rising stress wave, which allows gradual stress accumulation in the specimen and maintains the load at both ends of the specimen in an equilibrium state. The results show that the dynamic strength of three kinds of BD samples represented loading rates dependence, and FG sandstones are more sensitive for loading rates than MG and CG samples. Moreover, the peak strength is observed to increase linearly with an increasing stress rates, and the relationship between the dynamic BD strength and stress rates can be built through a linear equation. Finally, the failure modes of different grain sizes are discussed and explained by microfailure mechanism.
\end{abstract}

\section{Introduction}

In deep mining activities, impact loadings such as blasting and drilling can produce compressive stress wave, which is reflected as tensile wave at a free surface around the openings. The wave leads to the tensile failure of the deep surroundings since it is much weaker in tension than in compression. Therefore tensile failure as a major failure mode of rocks in underground rock engineering projects should be paid attention to as illustrated in Figure 1, where rocks are subjected to dynamic disturbances. As Figure 1 illustrated the tensile stress of rocks can be divided into two kinds of scales (macroscopic and microscopic scale). Microscopic tension can be mainly determined by the flaws that consisted of grain size of minerals and microcracks.

For years, significant progress has been made in the mechanical behavior of various types of geomaterials under high loading rates [1-3]. A considerable amount of work has been done on the effect of porosities [4], temperatures
[5], fracture toughness [6], loading rates [7], and pretension [8] regarding mechanical behaviors under dynamic loadings. Earlier researches have been conducted to obtain the tensile stress-strain curve with Brazilian disc (BD) under quasi-static loads [9]. The relationship between normalized dynamic tensile strength and loading rates has been discussed and results [10-13] show that the dynamic tensile sharply increases above a calculated value of critical strain rate $\left(10^{0} \mathrm{~s}^{-1}\right)$ and loading rate $\left(10^{2} \mathrm{GPa} / \mathrm{s}\right)$.

As we know, the rock microstructure has an obvious effect on the strength and failure model. The effects of presence of bedding or cleavage planes on rock strength were discussed, such as in Yule marble [14] and argillite [15]. Also the preferred orientation of fabric [16] or microstructure $[17,18]$ in granite affecting the mechanical behavior has been done. In addition, quantitative understanding of texture, orientation, and mineral content of the rock on anisotropy may contribute to better evaluation of the microstructure parameters $[6,19$, 20]. 


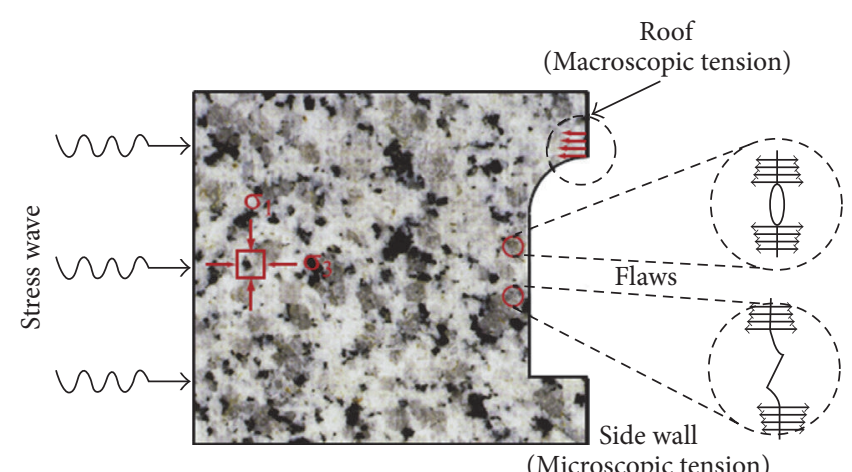

FIgURE 1: Potential sources of tensile failure around an underground opening [8].

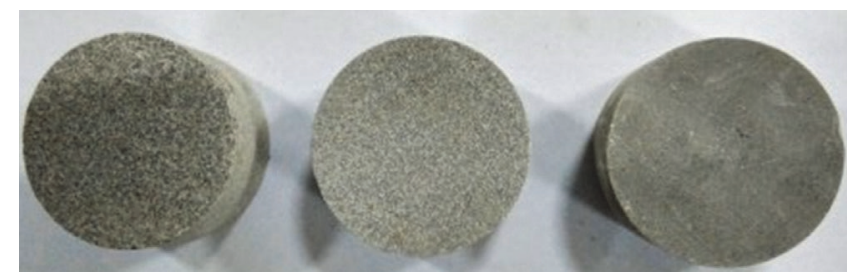

FIGURE 2: Three types of sandstones of Brazilian specimen.

Only a limited amount of work has been done on the effect of microstructure properties (e.g., mineral content, grain shape and distribution, grain size, and microcracks distribution) on rocks [21]. As many scientists and researchers thought that grain size plays a significant role in the macromechanical properties, many tests have been conducted under static loadings [22-24]. Also, it has been found by many researchers that the strength of rock decreases with increasing grain size $[25,26]$. This conclusion can be explained with Griffith theory in compression, as shown in [27]

$$
\sigma_{\mathrm{c}} \geq 8 \sqrt{\frac{2 E \alpha}{\pi c}},
$$

where $\sigma_{\mathrm{c}}$ is the compressive stress; $E$ is the elastic modulus; $\alpha$ is the surface energy of the crack surfaces; $c$ is the crack halflength and can be considered proportional to the grain size. Hence, greater grain sizes produce lower fracture compressive stresses.

The above researches and analysis can be summarized through the following conclusions: (1) few reports have been done regarding the Brazilian dynamic properties under immediate strain rate $s$; current studies focus on the dynamic tensile strength and fracture toughness under relatively smaller and higher strain rate, while ignoring the immediate strain rates $\left(10^{0} \sim 10^{2} \mathrm{~s}^{-1}\right)$ on the behavior of rock mechanical properties. (2) Many earlier researches primarily paid attention to the effect of physical changes (porosity, specimen size, and temperature) on rock mechanical properties under dynamic loadings but less attention to the relationship between microstructure parameters of rock (mineral content, grain distribution and size, etc.) and mechanical properties. (3) The above papers primarily considered the effect of grain size on the compressive strength and failure mode, but understanding on the change of Brazilian strength and failure mechanism is also important to the research of rock stability and safety control under dynamic disturbance.

In this paper, the experimental rock discs are drilled from the surrounding rocks in the field of Xiao Jihan Coals and processed to Brazilian disc. Furthermore, the grain sizes of sandstones can be determined by the depth of boreholes, which are grouped by three kinds of grain sizes (fine grain, medium grain, and coarse grain). Therefore the grain size as a key factor of microstructure can have mechanical influence and is taken into consideration. Mesostructure parameters analysis of the above samples can be obtained through optical microscopy, SEM, and X-ray diffraction. SHPB experimental system is conducted on three kinds of grain sizes of sandstones under different impact velocities loaded by pendulum hammer with the design of CRISR team in Northeastern University. In summary, the coupled effect of grain size and loading rate on the strength and failure modes of sandstones under dynamic loadings is discussed as follows.

\section{Sample Preparation and Testing Methodology}

\subsection{Sample Preparation}

2.1.1. Brazilian Samples of Sandstones and Petrographic Anal$y$ sis. The rock samples are three types of sandstones as illustrated in Figure 2 taken from the Xiao Jihan Coal Field of $\mathrm{Yu}$ Lin city in Shanxi province, located in the central region of China. Rock cores with a nominal diameter of $50 \mathrm{~mm}$ are drilled from the surrounding rocks in different mining levels. The samples of sandstones cannot be found the obvious 
TABLE 1: Basic parameters of the sandstones with different sizes.

\begin{tabular}{lccccccc}
\hline Types of sandstone & $\begin{array}{c}\text { Young's } \\
\text { modulus (GPa) }\end{array}$ & Poisson's ratio & $V_{P}(\mathrm{~m} / \mathrm{s})$ & $\begin{array}{c}\text { Compressive } \\
\text { strength } \\
(\mathrm{MPa})\end{array}$ & $\begin{array}{c}\text { Tensile } \\
\text { strength } \\
(\mathrm{MPa})\end{array}$ & $K_{\mathrm{IC}}\left(\mathrm{MPa} \cdot \mathrm{m}^{1 / 2}\right)$ & $\mathrm{Density}\left(\mathrm{kg} / \mathrm{m}^{3}\right)$ \\
\hline Coarse grain & 13.63 & 0.236 & 2501 & 48.6 & 3.99 & 0.52 & 2278 \\
Medium grain & 35.8 & 0.261 & 2658 & 62.57 & 5.8 & 0.58 & 2354 \\
Fine grain & 53 & 0.285 & 2899 & 123.56 & 12.62 & 0.73 & 2700 \\
\hline
\end{tabular}

TABLE 2: Mineral compositions of the three types of sandstone.

\begin{tabular}{lccc}
\hline Mineral & \multicolumn{3}{c}{ Mass percentage } \\
& CG & MG & FG \\
\hline kaolinite & 12 & 10 & 8 \\
Quartz & 50 & 40 & 55 \\
Plagioclase & 20 & 25 & 22 \\
K-feldspar & 18 & 25 & 15 \\
\hline
\end{tabular}

bedding layers and thus they are regarded intact from the macroscopic scale. Special care is taken to prepare the Brazilian disc specimens with a diameter of $50 \mathrm{~mm}$ and thickness of $25 \mathrm{~mm}$ and standard specimens with a diameter of $50 \mathrm{~mm}$ and thickness of $50 \mathrm{~mm}$. The samples drilled from the surrounding rocks can be grouped by three types, which are named fine grained, medium grained, and coarse grained, abbreviated FG, MG, and CG. Details of the rock characterization are shown in Table 1, which summarizes the physical and mechanical properties of the specimen.

Thin sections produced from each type of sandstone are analyzed by a polarized microscope, with determination of the grain size distribution. Figures 3 and 4 show typical microscopic images of thin sections and the corresponding grain size distributions of the three types of sandstones, respectively. Figure 4 represents the percentage of different grain sizes in each kind of sandstone and moreover it can be calculated that mean grain sizes of FG, MG, and CG sandstones are 30,105 , and $231 \mu \mathrm{m}$, respectively, which shows clear differences among the three types of sandstones.

Mineralogical content is obtained from the studied rocks with an X-ray diffraction test illustrated in Figure 5. The samples show similar features in terms of main constituent minerals (Table 2), that is, quartz, plagioclase, $\mathrm{K}$-feldspar, and minor clay minerals (kaolinite). According to the mineralogy, it is clear that CG sandstone contains the highest and lowest percentage of quartz and clay minerals, respectively, while FG sandstone shows the lowest quartz content and highest content of clay minerals.

\subsection{Experimental Apparatus and Testing Methodology}

2.2.1. Experimental Apparatus. As shown in Figure 6 schematically, the pendulum hammer-driven SHPB apparatus possesses three important components: a loading device, bar components, and a data acquisition and recording system. We adopt the pendulum hammer as a loading device to generate the impact stress wave (half-sine wave). Bar components are composed of an incident bar, a transmission bar, and a momentum trap device at the end. Between the incident bar and the transmission bar the rock specimen is sandwiched. The incident and transmission bars are both $2.0 \mathrm{~m}$ in length. The data acquisition and recording system is made up of a preamplifier, a digital oscilloscope, and a microcomputer. The digital oscilloscope rate is 10 million Bps; the recording system bandwidth is $100 \mathrm{kHz}$; the data precision is 12 bits. Data acquisition and recording system coheres with the proposed method of International Society for Rock Mechanics (ISRM) for dynamic Brazilian test. And the variation of the swing angle of the pendulum hammer controls the velocity of hammer impacting incident bar and shape of incident wave. The incident strain and reflected strain are measured by strain gauges on incident bar, and transmitted strain is measured on transmission bar. During SHPB experiment, we can use them to calculate the force at both ends of rock specimen.

The impact velocities are originated from the different swing angles of pendulum hammer. In this paper, four swing angles of $40^{\circ}, 50^{\circ}, 70^{\circ}$, and $90^{\circ}$ are set under dynamic experiments corresponding to the four impact velocities of $2.0 \mathrm{~m} / \mathrm{s}$, $2.5 \mathrm{~m} / \mathrm{s}, 3.3 \mathrm{~m} / \mathrm{s}$, and $4.2 \mathrm{~m} / \mathrm{s}$. Moreover, the swing angle of $40^{\circ}$ is the critical value to make the Brazilian specimens fail. To be specific, when the impact velocity is lower than $2.0 \mathrm{~m} / \mathrm{s}$, the sandstones show a complete status and cannot cause the failure.

2.2.2. Testing Methodology. International Society for Rock Mechanics issued the suggested methods on dynamic Brazilian test for determining the dynamic tensile strength and fracture toughness of rock materials. The rock specimen is impacted in radial direction and the loading condition is shown in Figure 7. In this respect, compression stress and strain and particle velocity to the right are regarded as the positive direction in the figure. Interfaces I and II are represented to the contact between the specimen and bars.

There are two fundamental assumptions on the conventional SHPB experimental technique. One is dynamic stress equilibrium. The stress at the contact area between rock disc and incident and transmission bars becomes equalized, after the stress wave propagates and is reflected several times, if the length of rock specimen is much less than wave length of the incident stress wave. The second is the one-dimensional elastic stress wave theory is valid when the stress wave propagates in both the incident and transmission bars without dispersion. During the SHPB experiment, the contact forces between rock specimen and incident and transmission bar 

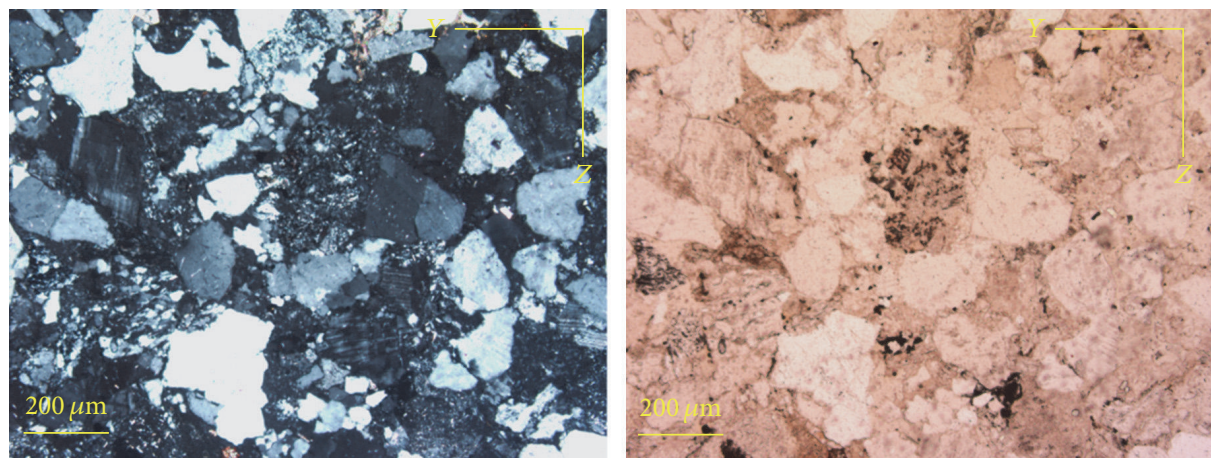

(a)
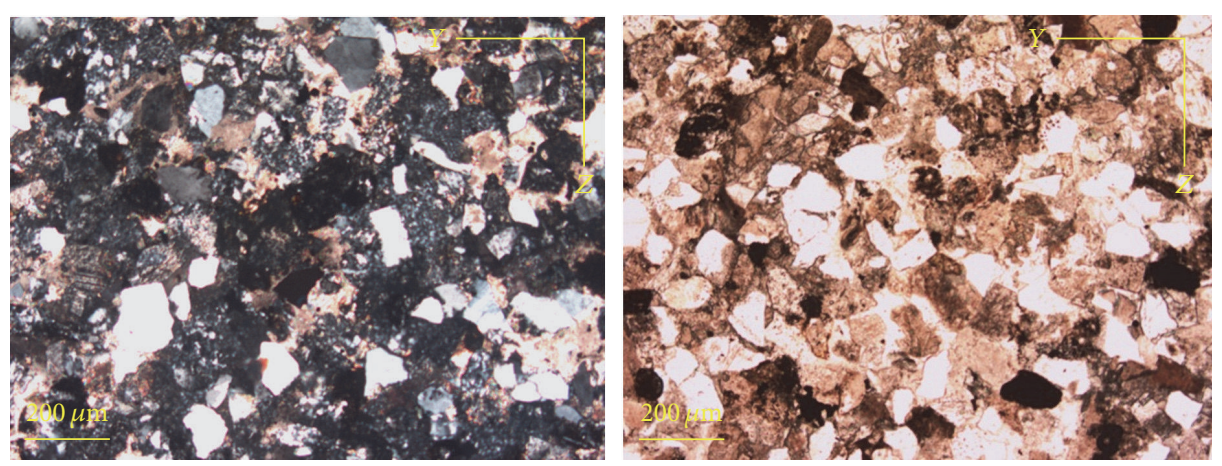

(b)
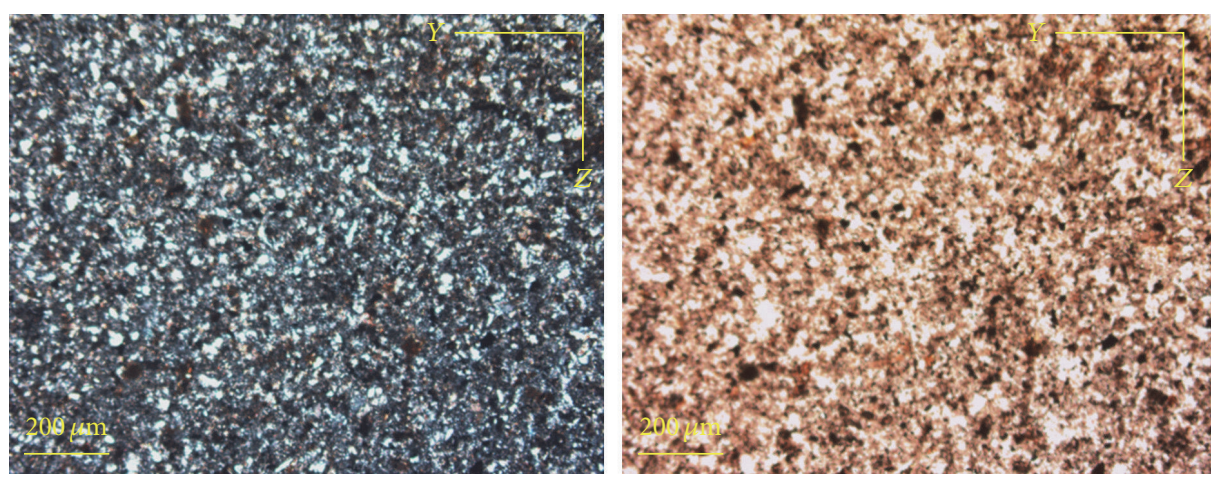

(c)

FIGURE 3: Microscopic images of thin sections and corresponding grain size distribution of the three types of sandstones.

(interfaces I-I and interfaces II-II) are denoted with $P_{1}(t)$ and $P_{2}(t)$, which can be calculated as

$$
\begin{aligned}
& P_{1}(t)=A E\left[\varepsilon_{I}(t)+\varepsilon_{R}(t)\right], \\
& P_{2}(t)=A E \varepsilon_{T}(t),
\end{aligned}
$$

where $A$ and $E$ are the cross-sectional area and Young's modulus of bars, respectively; the incident, reflected, and transmitted strains, $\varepsilon_{I}, \varepsilon_{R}$ and $\varepsilon_{T}$, are measured by the strain gauges on the incident bar and transmission bar.

The stress field reflects back and forth increasing in magnitude, and the specimen remains in equilibrium until the time of failure, once the specimen is in equilibrium. The Brazilian static stress relationship is also applied to calculate the dynamic tensile strength of a specimen loaded in the
SHPB test. This principle is also applicable for the pendulum hammer-driven SHPB test as proposed in this study. Consequently, the tensile stress at the center of the Brazilian disc specimen can be calculated as

$$
\sigma(t)=\frac{P_{1}(t)+P_{2}(t)}{\pi D H}=\frac{A E\left(\varepsilon_{I}(t)+\varepsilon_{R}(t)+\varepsilon_{T}(t)\right)}{\pi D H} .
$$

\section{Dynamic Brazilian Test and Results}

Dynamic Brazilian tests are carried out by means of the pendulum hammer-driven SHPB apparatus. The sandstone specimens (three kinds of grain sizes) are 50 in diameter and $25 \mathrm{~mm}$ in thickness. The cylindrical surfaces have no signs of obvious tool marks. End faces flat to $0.25 \mathrm{~mm}$ are parallel to within $0.25^{\circ}$. The incident bar with pendulum hammer is 




FIgURE 4: Initial grain size distribution of the granular sample.
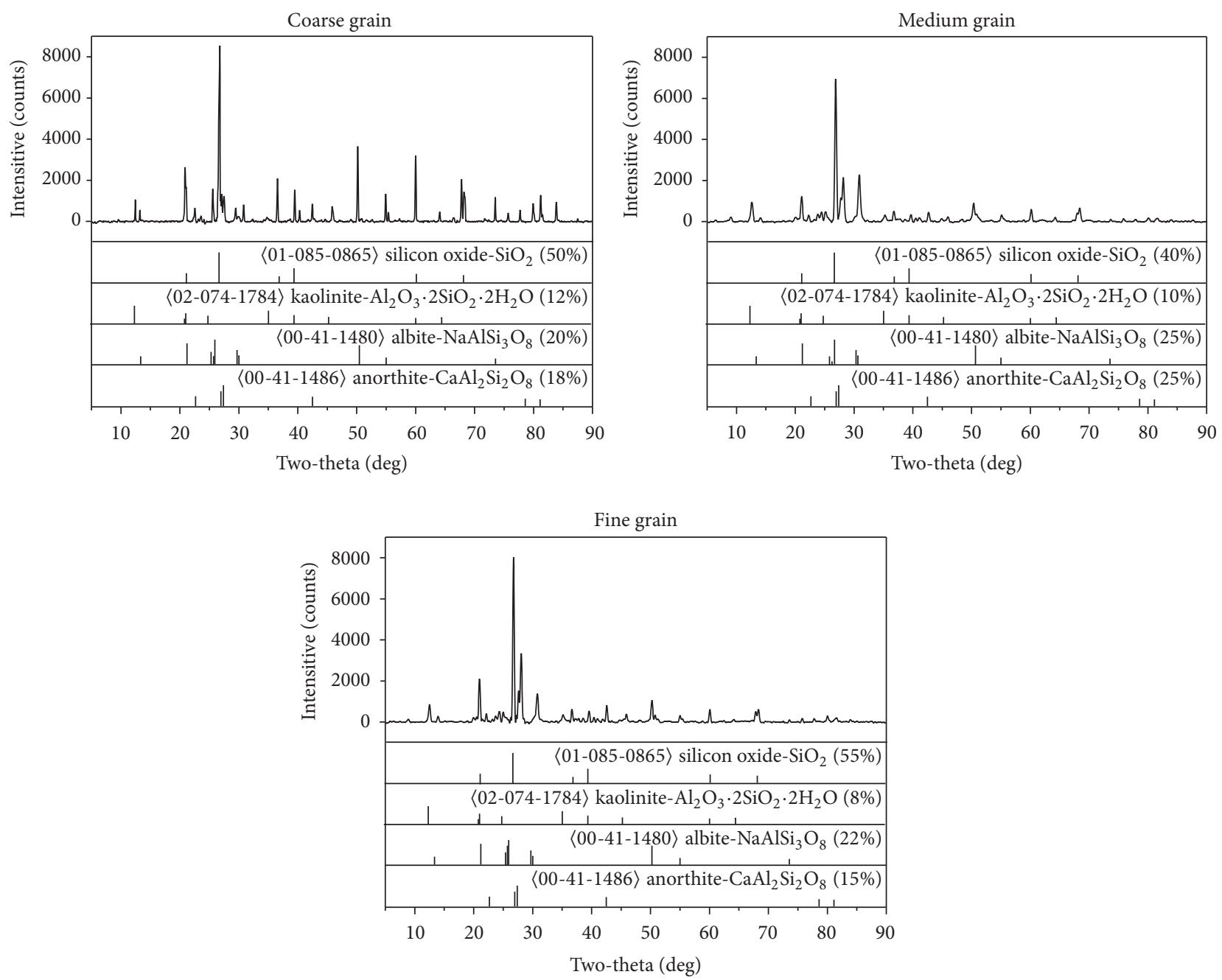

FIGURE 5: XRD graphs of sandstones with three kinds of grain sizes. 


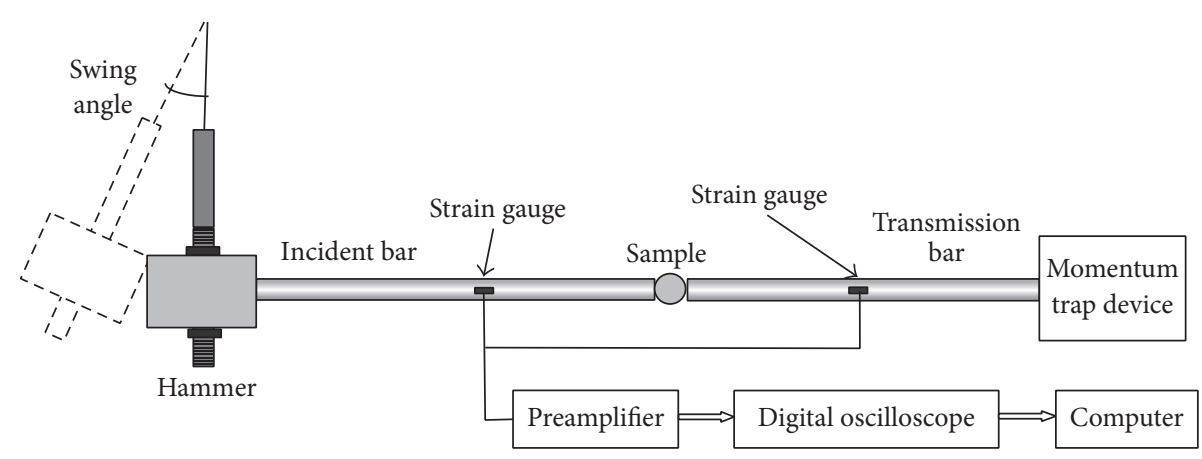

FIgURE 6: The pendulum hammer-driven SHPB experimental apparatus.

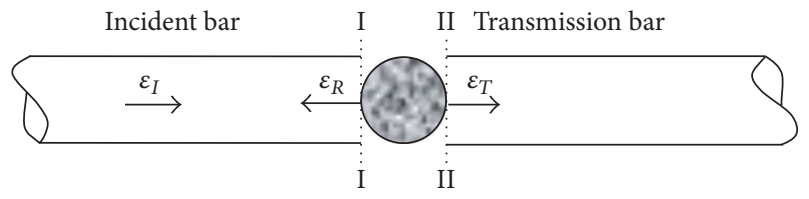

FIGURE 7: Wave propagation between bar and specimen interfaces.

impacted under different velocities, which generates the incident waves presented in Figure $8(\mathrm{a})(V=2.0 \mathrm{~m} / \mathrm{s}, 2.5 \mathrm{~m} / \mathrm{s}$, $3.3 \mathrm{~m} / \mathrm{s}$, and $4.2 \mathrm{~m} / \mathrm{s}$ ), and thus the incident stress waves could be regulated through accurate specification of the swing angle. In this research, three kinds of sandstones grouped are tested under different impact velocities of 2.0, 2.5, 3.3, and $4.2 \mathrm{~m} / \mathrm{s}$, in accordance with the loading rates of, $391,542,741$, and $933 \mathrm{GPa} / \mathrm{s}$, which can be calculated by the division of peak stress of incident stress waves and corresponding time in Figure 8(a) shown.

It can be seen from Figure 8(a) that the incident wave produced by pendulum hammer is considered as the ideal pulse shape to eliminate the effect of wave dispersion and to achieve a constant in the rock specimen. Therefore, compared with the conventional SHPB test apparatus, it is not requisite to use the pulse shaper to attain this type of ideal incident waveform. From Figure 8(b), constant can be obtained by pasting the strain gauges in the middle and two ends of Brazilian samples under dynamic loadings during the time period between $120 \mu \mathrm{s}$ and $280 \mu \mathrm{s}$, which guarantees the validity of the test results.

3.1. Data Reduction and Dynamic Force Balance. Figure 9 shows the signals of the relationship between time and voltage captured by the strain gauges on the bars. The red line represents the signals of the incident wave and reflected wave, while the black line delegates the signal of the transmitted wave. The motion induced by the incident wave is to the right and thus the flange has no effect on the wave propagation. The strains of incident wave, reflected wave, and transmitted wave are denoted by $\varepsilon_{I}, \varepsilon_{R}$, and $\varepsilon_{T}$, as the following equation mentioned, respectively.

Earlier researches on static and dynamic BD tests have all proven that the $\mathrm{BD}$ test can obtain accurate strength results once the stress force equilibrium should be satisfied before the specimen fails. Figure 10 shows the dynamic force balance at the end of Brazilian samples recorded by the strain gauges attached on the incident bar and the transmitted bar, respectively. The force on one side of the incident bar is the sum of forces by the incident and reflected stress waves marked as In + Re, while on the other side of the bar it is by the transmitted stress wave marked as Tr. In the figure it is well known that the dynamic forces on both sides of the samples are identical during the loading period, which can prove the accuracy of the experiment.

3.2. Effect of Stress Rate. Three kinds of grain sizes were grouped and tested by different impact velocities of $V=$ $2.0 \mathrm{~m} / \mathrm{s}, 2.5 \mathrm{~m} / \mathrm{s}, 3.3 \mathrm{~m} / \mathrm{s}$, and $4.2 \mathrm{~m} / \mathrm{s}$. Results are listed in Table 3, and the dynamic tensile strength is the maximum value of the stress-time curve as Figure 11 illustrated.

In this study, the major factors affecting the strength of the sandstones are considered in this paper, which are loading rates and grain sizes. According to (3), $\varepsilon_{I}, \varepsilon_{R}$, and $\varepsilon_{T}$ are used to calculate the dynamic tensile strength $\sigma_{t}$ at the center of the specimen, of which stress is perpendicular to the loading direction. As shown in Table 3, the strength of sandstones of fine grain size is much higher than that of medium-grain size and coarse grain size. Figure 11 shows the relationship between tensile stress and corresponding duration time in the center of sandstones.

The stress rate can be calculated by (5), in which $\sigma_{d t}$ and $t$ represent the dynamic tensile strength of sandstones and duration of peak stress reached as Figure 11 shows, respectively. The dynamic Brazilian test has been conducted by Zhu et al. [28] to investigate the dynamic tensile characteristic using SHPB experiment combined with numerical simulation. The stress rates of the Brazilian samples range from $80 \mathrm{GPa} / \mathrm{s}$ to $250 \mathrm{GPa} / \mathrm{s}$ and the tensile strength is 9 $16 \mathrm{MPa}$. Yao et al. [29] has used Longyou sandstones to study the coupled effect of the loading rates and temperature on the mechanical behaviors under dynamic impacts. Moreover the stress rates stay in $20 \sim 600 \mathrm{GPa} / \mathrm{s}$, and the dynamic tensile strength of samples is also between $3 \mathrm{MPa}$ and $16 \mathrm{MPa}$. Liao et al. [30] used the combined methods to study the dynamic mechanical properties of sandstones and the results show that 


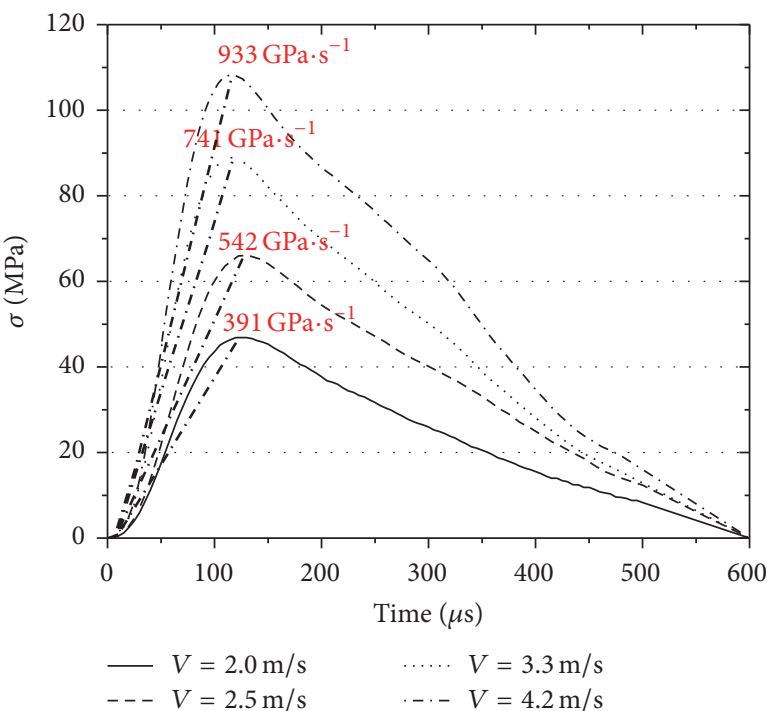

(a)

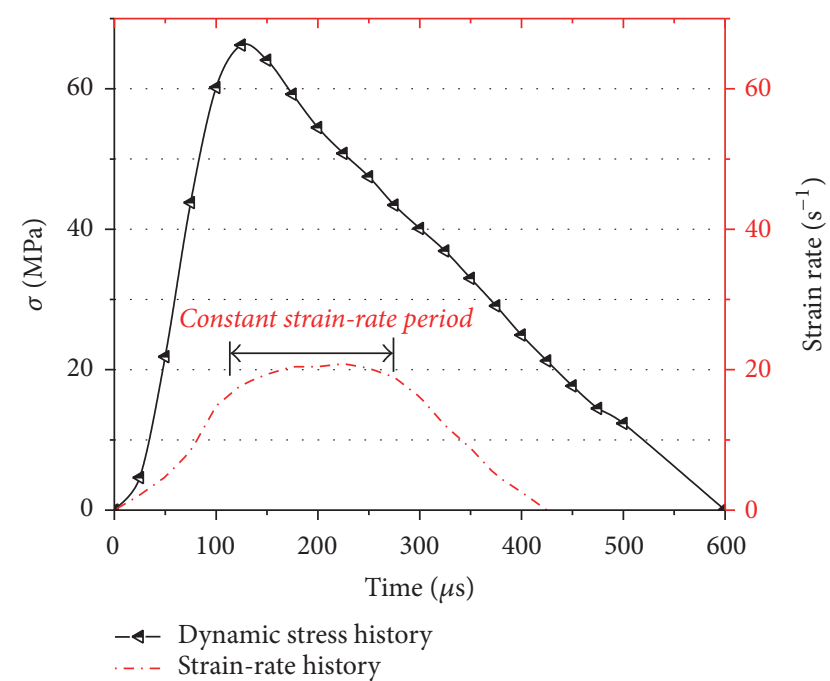

(b)

FIGURE 8: (a) Measured incident stress waves under different impact loadings and (b) complete strain-rate curve under certain velocities $(V=2.5 \mathrm{~m} / \mathrm{s})$.

TABLE 3: Summary of dynamic tensile test results with different grain sizes.

\begin{tabular}{|c|c|c|c|c|c|c|}
\hline Types of sandst & velocity $(\mathrm{m} / \mathrm{s})$ & Specimen number & Diameter (mm) & Thickness (mm) & Stress rate $(\mathrm{GPa} / \mathrm{s})$ & $\begin{array}{l}\text { Dynamic tensile } \\
\text { strength }(\mathrm{MPa})\end{array}$ \\
\hline \multirow{4}{*}{ Coarse grain } & 2.0 & $\mathrm{C}-1$ & 49.98 & 24.97 & 25.7 & 5.34 \\
\hline & 2.5 & C-2 & 49.88 & 24.99 & 65.5 & 7.57 \\
\hline & 3.3 & C-3 & 50.12 & 25.03 & 125.4 & 10.23 \\
\hline & 4.2 & C-4 & 50.23 & 25.12 & 180.7 & 13.75 \\
\hline \multirow{4}{*}{ Medium grain } & 2.0 & M-1 & 48.73 & 24.87 & 62.5 & 10.4 \\
\hline & 2.5 & $\mathrm{M}-2$ & 49.23 & 25.23 & 88.2 & 13.6 \\
\hline & 3.3 & M-3 & 49.87 & 24.96 & 126.5 & 17.8 \\
\hline & 4.2 & M-4 & 50.24 & 24.98 & 176.7 & 22.5 \\
\hline \multirow{4}{*}{ Fine grain } & 2.0 & F-1 & 49.99 & 25.01 & 86.8 & 14.3 \\
\hline & 2.5 & F-2 & 48.94 & 25.14 & 115.7 & 19.5 \\
\hline & 3.3 & F-3 & 51.22 & 24.99 & 227.5 & 29.8 \\
\hline & 4.2 & F-4 & 49.87 & 24.98 & 328.6 & 36.5 \\
\hline
\end{tabular}

the dynamic strength increases from 13 to $20 \mathrm{MPa}$ with the increase of the loading rates. The similar conclusion drawn by $\mathrm{Wu}$ et al. [8] shows that the loading rates vary from 190 to $990 \mathrm{GPa} / \mathrm{s}$ and the dynamic tensile strength values are between $20 \mathrm{MPa}$ and $40 \mathrm{MPa}$ for granite samples. Therefore, the stress rates used in this paper are reasonable. It is noticed that the loading rates mentioned in the above papers are referred to as stress rates in this paper and the comparison of the results can be found in Figure 12.

$$
\dot{\sigma}=\frac{\sigma_{\mathrm{dt}}}{t}
$$

3.3. Effect of Grain Size. It is found that the dynamic tensile strength is larger than the static tensile strength of $3.99,5.8$, and $12.62 \mathrm{MPa}$ (representing CG, MG, and FG). In particular, the dynamic tensile strength increases with the rising impact velocity of the pendulum hammer, thus confirming the loading rate dependency of tensile strength of the samples. Figure 13 depicts an obvious growth in peak strength with increasing impact velocities for CG, MG, and CG sandstone, which can be measured by the dynamic increase factor (DIF) that is calculated by the division of dynamic strength and static strength of sandstones. Moreover it can be found from the figure that the values of DIF for FG are bigger than that for CG and MG, and therefore the dependency is stronger for FG than that for MG and CG.

FG sandstone is the most responsive to loading rates compared to MG and CG sandstones, which shows a steeper 


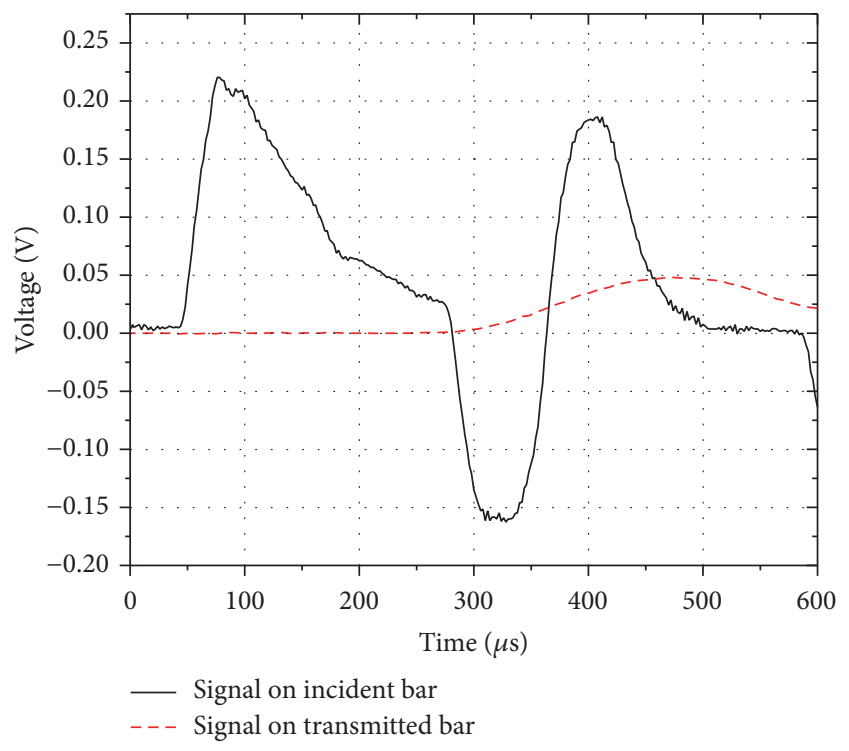

Figure 9: Original signals in a typical dynamic Brazilian test.

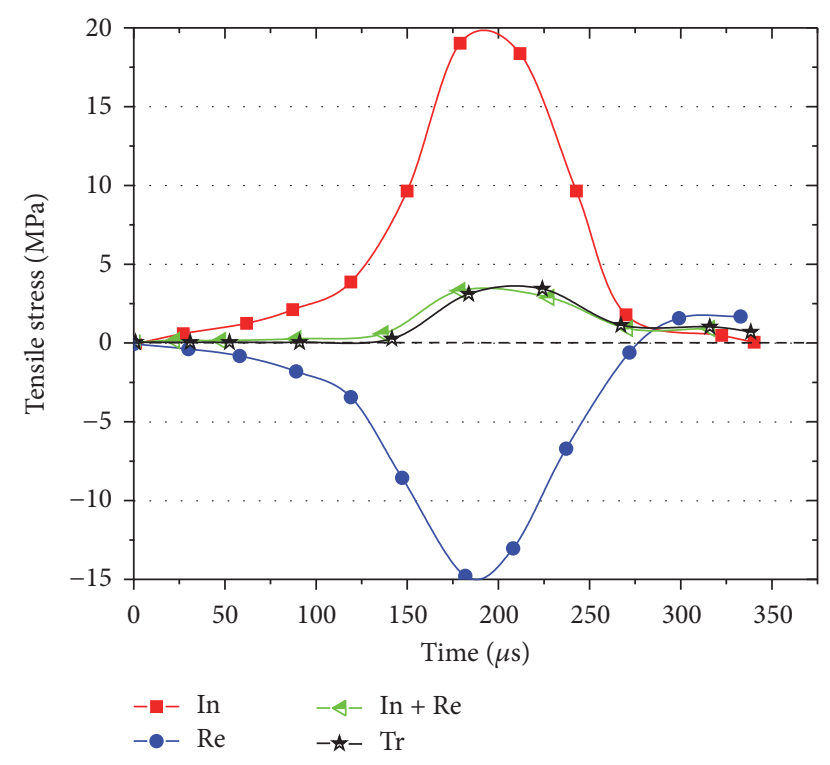

FIgURE 10: Dynamic force balance of Brazilian samples $(V=$ $3.3 \mathrm{~m} / \mathrm{s}$ ) in the SHPB test.

increase of peak strength under different impact velocities. This phenomenon is perhaps mainly resulting from FG sandstone with the relatively smaller grain size, whose microcracks, developing during dynamic loadings, are much smaller and more scattered, as the grains are finer. Thus, the applied stress is inclined to disperse within the specimen. This applied stress in greater diffusion causes that the rock specimen at higher loading rates can stiffen much further and bring about greater strength increase compared with medium and coarser grain sandstones.

3.4. Data Fitting of the Results. Figure 14 shows a linear increase in peak strength with increasing stress rates for three kinds of sandstones. The relationships of dynamic tensile strength and stress rates for CG, MG, and FG are represented in (6), (7), and (8), respectively.

$$
\begin{array}{ll}
\sigma_{\mathrm{dt}}=0.0328 \dot{\sigma}+3.75 & \left(R^{2}=0.9797\right), \\
\sigma_{\mathrm{dt}}=0.0648 \dot{\sigma}+4.99 & \left(R^{2}=0.9782\right), \\
\sigma_{\mathrm{dt}}=0.0908 \dot{\sigma}+11.96 & \left(R^{2}=0.9898\right) .
\end{array}
$$

According to the testing result, it is interestingly found that the intercept of linear fitting is equal to the value of static tensile strength of three kinds of grain sizes. Therefore relationships of dynamic tensile strength and stress rates can be represented by

$$
\sigma_{\mathrm{dt}}=\mu \dot{\sigma}+\sigma_{\mathrm{t}}
$$

where $\sigma_{\mathrm{dt}}$ is the dynamic tensile strength; $\dot{\sigma}$ is the stress rate; $\sigma_{\mathrm{t}}$ is the static tensile strength; $\mu$ is fitting parameter. Figure 14 is obtained to show how the dynamic tensile strength increases with the stress rates and grain sizes, which fits well the data points.

3.5. Discussion of the Results. It is interesting that the results show the rate-sensitivity of fine grain can be higher than that of coarse grain sample. At mesoscale, rock specimen is considered as a heterogeneous and composite material consisting of different grain sizes and cement. Many earlier researches have been made to demonstrate that increase of the rock heterogeneity contributes to an increase in the strain-rate dependency [31]. But, in this paper, the effect of heterogeneity on strain-rate sensitivity is not taken into consideration, and another mechanism which may influence the strength change is grain size inertia effect. As Figure 1 illustrated,

$$
\sigma_{z}=\frac{K_{\mathrm{IC}}}{\sqrt{\pi c}}
$$

where $K_{\text {IC }}$ represents quasi-static fracture toughness of crack tips and $\sigma_{z}$ is the external tensile stress. In this paper grain size $2 C$ of rock is equal to crack length quantitatively. Equation (11) took the crack interaction into consideration and ultimate uniaxial tensile strength $\sigma_{\text {ts }}$ can be obtained:

$$
\sigma_{\mathrm{ts}}=K_{\mathrm{IC}}\left(2 b \tan \frac{\pi c}{2 b}\right)^{-0.5}
$$

where $2 b$ is the grain spacing; Mode-I dynamic stress intensity factor of a crack with an instantaneous growth speed $\dot{l}(t)$ of the crack can be equal to stationary crack array at the same instantaneous crack length $l(t)$ :

$$
\begin{gathered}
K_{\mathrm{Id}}=k(i) K_{\mathrm{I}}^{\text {array }}, \\
K_{\mathrm{I}}^{\text {array }}=\sigma_{z} \sqrt{2 b \tan \left(\frac{\pi l}{2 b}\right)},
\end{gathered}
$$

where $k(i)$ is introduced to represent the grain size (equal to crack) inertia effect on the stress intensity factor (SIF) during 

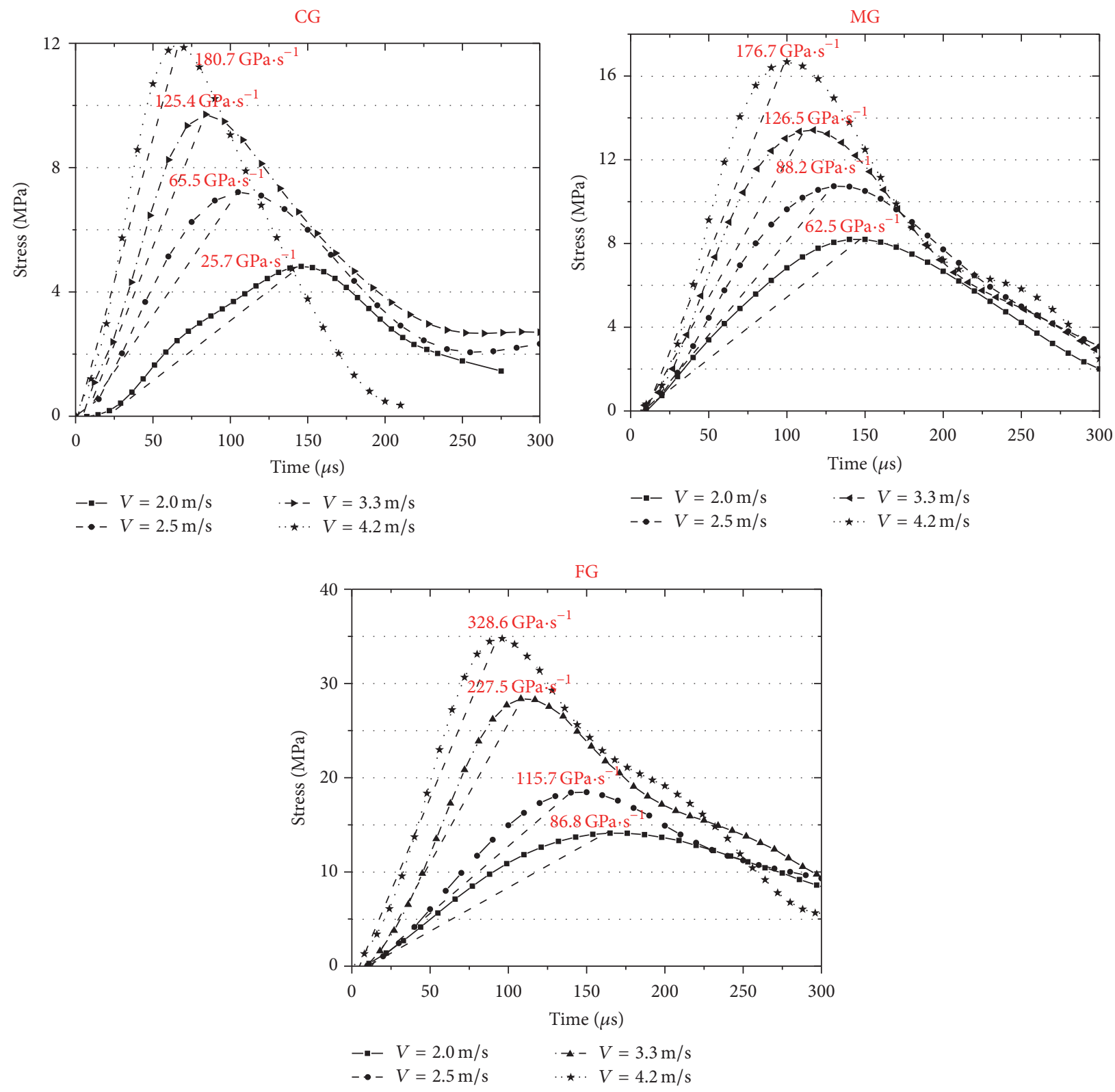

FIGURE 11: The time history of tensile stress at the center of Brazilian disc sample of three kinds of sandstones.

dynamic crack growth. And $k(i)$ can be expressed by the following equation [32]:

$$
\begin{aligned}
k(i) & =\frac{C_{r}-\dot{i}}{C_{r}-0.5 i}, \\
C_{r} & =\frac{0.862+1.14 V_{s}}{1+V_{s}} \sqrt{\frac{E_{s}}{2\left(1+V_{s}\right) \rho_{s}}},
\end{aligned}
$$

in which $C_{r}$ is the speed of Rayleigh wave and $V_{s}$ and $\rho_{s}$ are the Poisson's ratio and material density of the rock.

According to the crack growth criterion, cracks would undergo growth when $K_{\text {Id }}$ reached the dynamic fracture toughness, which as a material constant is not changed with strain-rate $\left(K_{\mathrm{IC}}=\right.$ constant $)$. Therefore the dynamic crack growth criterion was expressed:

$$
K_{\mathrm{IC}}=K_{\mathrm{Id}}^{\text {array }} .
$$

Then, combining (13)-(14), the crack growth speed can be represented:

$$
i=C_{r} \frac{K_{\mathrm{I}}^{\text {array }}-K_{\mathrm{IC}}}{K_{\mathrm{I}}^{\text {array }}-0.5 K_{\mathrm{IC}}} .
$$

The crack length is calculated by integrating the crack growth speed until failure happened. $t_{f}$ is the failure time and connected with the situation that $l$ is equal to $b$.

$$
b=\int_{0}^{t_{f}} i d t
$$




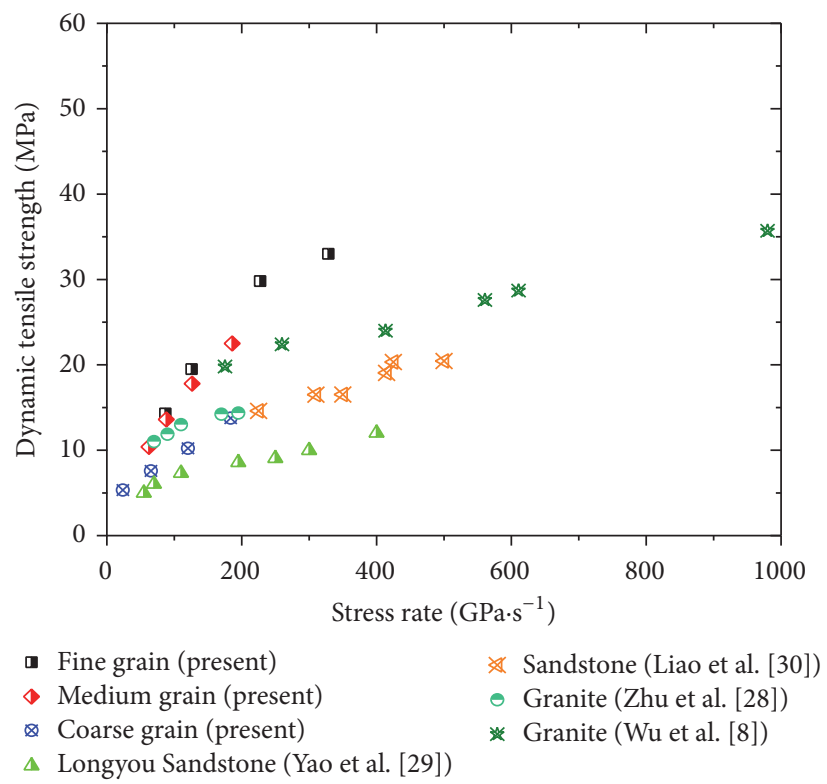

FIGURE 12: Dynamic Brazilian results obtained in this paper and their comparison to the existing results.
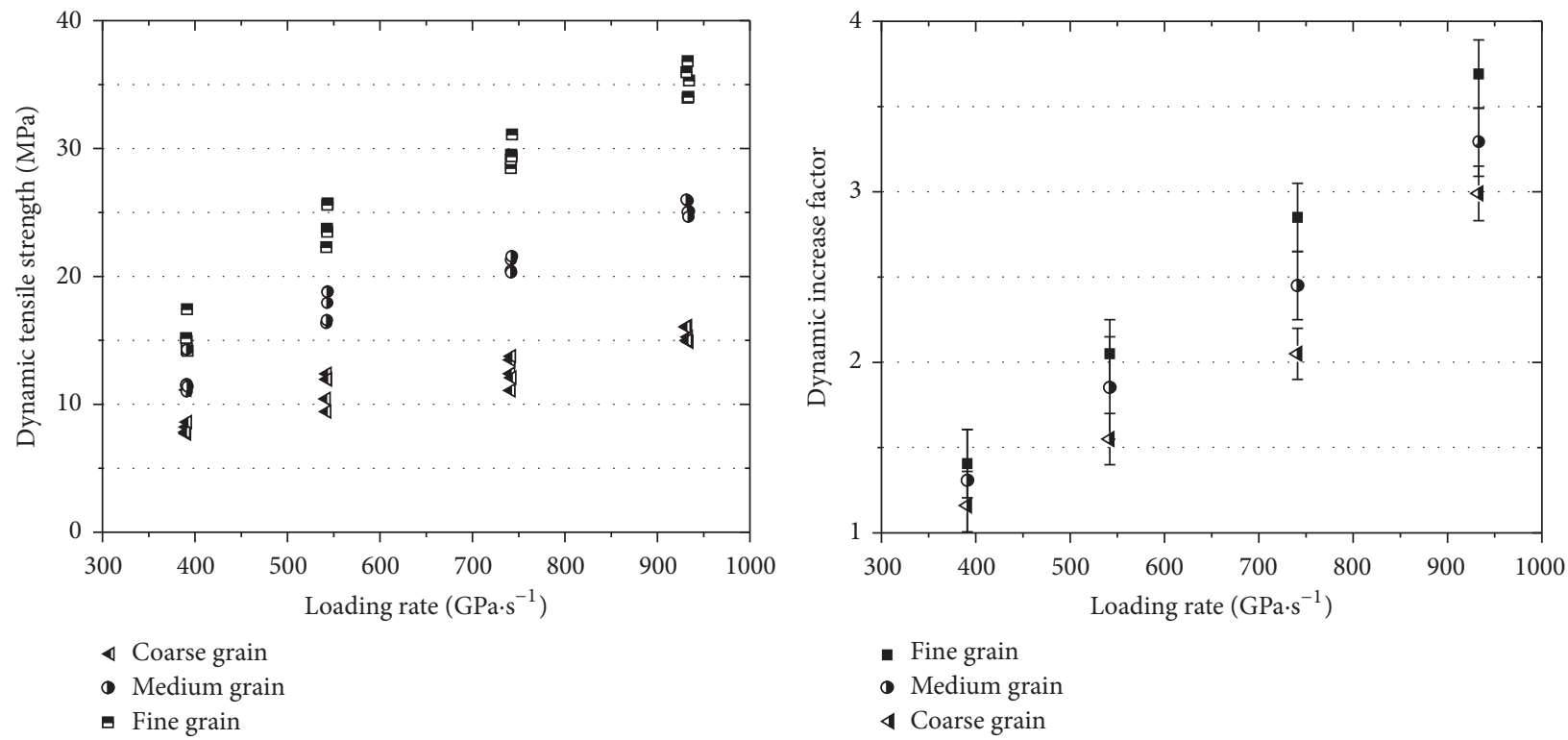

FIGURE 13: The relationship of loading rate dependence and dynamic tensile strength increase index of three kinds of grain sizes.

Finally, the DIF of dynamic tensile strength can be deduced from the equation below, where $\sigma_{\text {ts }}$ is the static tensile strength of rock samples.

$$
\mathrm{DIF}=\frac{\sigma_{z}\left(t_{f}\right)}{\sigma_{\mathrm{ts}}} .
$$

In this paper, three kinds of sandstones with different grain sizes are illustrated. The parameters of the samples are found from Table 1 and the model is applied to test the results from Figure 13, which represents the tensile strength plotted as a function of the stress rate and strain dependency with the change of loading rates. It shows that DIF increases with the decrease of the grain size in the medium strain regions, which implies that grain inertia theory is one of the mechanisms responsible for the strain-rate sensitivity of the tensile strength of rock materials.

3.6. Failure Mechanism. According to the general principle of Brazilian test, the cracks initiate at the center of the Brazilian disc specimen caused by tensile stress and growth to the two sides of specimen, thus dividing the specimen into two halves. Figure 15 illustrates the postfailure characteristics of Brazilian specimens with different grain sizes tested under 

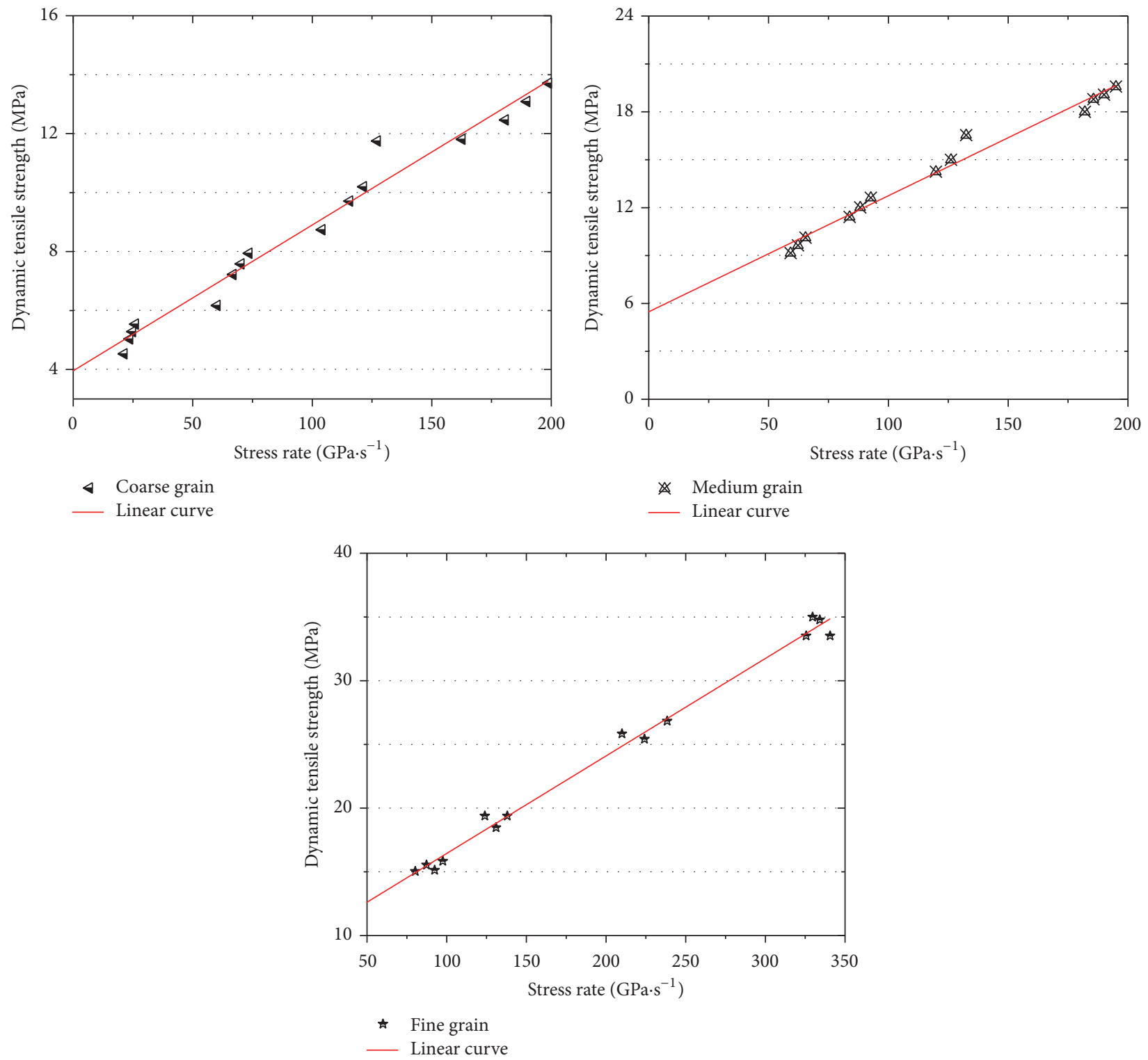

FIGURE 14: The dynamic strength versus stress rates for sandstones with different grain sizes.

different impact velocities. The failure mode is separated into two halves, when impact velocity is lower; the secondary vshaped failure zone is generally observed near the contact area between bars and the specimens, as impact velocity is relatively higher. But, in terms of FG sandstone, the failure is dominated by shearing mode, and a considerable amount of tensile failure (in the center of specimen) has taken place throughout the samples under higher loading rates and formed tensile failure zone.

\section{Conclusions}

This paper picks sandstones from Xiao Jihan Coal processed to Brazilian disc and uses the pendulum hammer-driven SHPB system to study the effect of grain size on the mechanical behaviors under different impact velocities.
The result reveals that three kinds of Brazilian disc are enhanced in tensile strength with the increase of the loading rates, which show the rate dependency for many rock materials. To be specific, FG sandstone is most sensitive to loading rates, which has a maximum value of dynamic increase factor, while MG and CG samples show a relatively lower value and dynamic tensile strength. Moreover, a model is introduced to explain the rate dependence of Brazilian disc with different grain sizes. Another important finding is samples with different grain sizes that represented different failure modes. With the rising impact velocities, each sample shows an increasing failure zone caused by tensile and shear stress, while the fine-grained samples represent a more obvious coupled failure model compared to medium and coarse grained samples. 


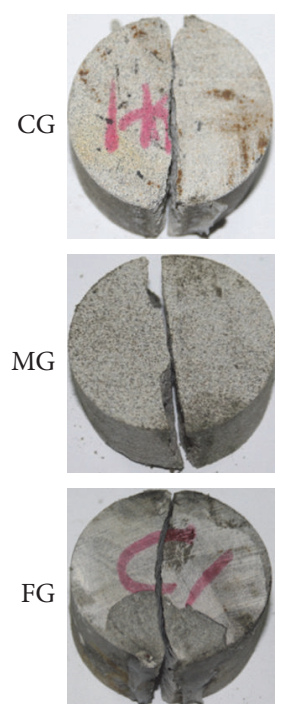

(a) $V=2.0 \mathrm{~m} / \mathrm{s}$

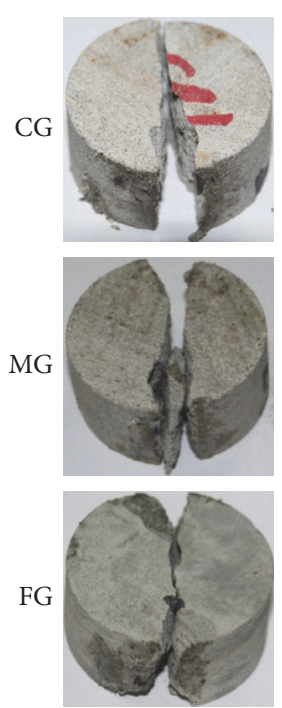

(b) $V=2.5 \mathrm{~m} / \mathrm{s}$

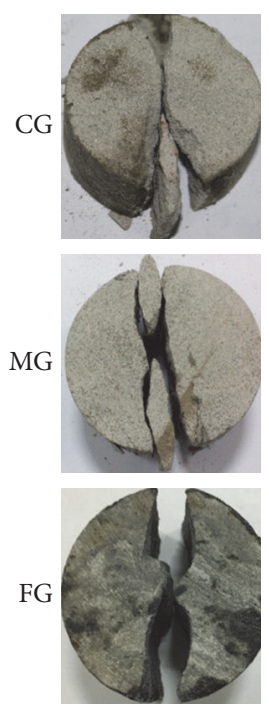

(c) $V=3.3 \mathrm{~m} / \mathrm{s}$

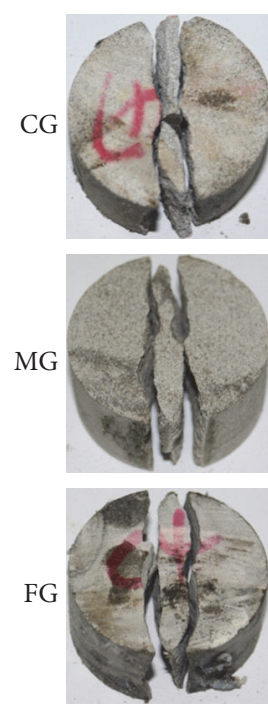

(d) $V=4.2 \mathrm{~m} / \mathrm{s}$

FIGURE 15: Failure modes of sandstone samples during dynamic Brazilian tests under different loading rates.

The damage and failure of surrounding rock are closely related to the rock microstructure and stress wave induced by the drilling and blasting in mining. Therefore, the coupled effect of loading rates and grain size on dynamic tensile strength of sandstones is discussed in this paper. Although it is mainly mechanism study, the results can also be used to provide a theoretical foundation for optimizing the parameters of the explosive during blasting process in different mining zones [33-35]. On the other hand, the support types of tunnel are influenced by the external disturbance and structure. So the results can also be attributed to decide the support types aiming to surrounding rocks in different levels, which can be an effective way to optimize the support type based on the analysis of mechanical mechanism under dynamic loadings [36, 37]. To be specific, when the surrounding rocks are mainly made of sandstones with fine grain sizes, lesser charge weight might be needed in order to excavate the same volume of ore rocks, and moreover the supporting structures might be needed consolidated because those with fine grain sizes are more vulnerable to dynamic loads.

\section{Conflicts of Interest}

The authors declare that they have no conflicts of interest.

\section{Acknowledgments}

This work was funded by the National Science Foundation of China (Grant nos. 51525402 and 51304037) and the Fundamental Research Funds for the Central Universities (Grant nos. N160104008 and N160103005).

\section{References}

[1] R. Chen, K. Xia, F. Dai, F. Lu, and S. N. Luo, "Determination of dynamic fracture parameters using a semi-circular bend technique in split Hopkinson pressure bar testing," Engineering Fracture Mechanics, vol. 76, no. 9, pp. 1268-1276, 2009.

[2] F. Dai, R. Chen, and K. Xia, "A Semi-circular bend technique for determining dynamic fracture toughness," Experimental Mechanics, vol. 50, no. 6, pp. 783-791, 2010.

[3] F. Dai, K. Xia, J. P. Zuo, R. Zhang, and N. W. Xu, "Static and dynamic flexural strength anisotropy of barre granite," Rock Mechanics and Rock Engineering, vol. 46, no. 6, pp. 1589-1602, 2013.

[4] E. Kim and H. Changani, "Effect of water saturation and loading rate on the mechanical properties of Red and Buff Sandstones," International Journal of Rock Mechanics and Mining Sciences, vol. 88, pp. 23-28, 2016.

[5] T. Yin, X. Li, K. Xia, and S. Huang, "Effect of thermal treatment on the dynamic fracture toughness of laurentian granite," Rock Mechanics and Rock Engineering, vol. 45, no. 6, pp. 1087-1094, 2012.

[6] Q. B. Zhang and J. Zhao, "Effect of loading rate on fracture toughness and failure micromechanisms in marble," Engineering Fracture Mechanics, vol. 102, pp. 288-309, 2013.

[7] M. D. Kuruppu, Y. Obara, M. R. Ayatollahi, K. P. Chong, and T. Funatsu, "ISRM-suggested method for determining the mode I static fracture toughness using semi-circular bend specimen," Rock Mechanics and Rock Engineering, vol. 47, no. 1, pp. 267-274, 2014.

[8] B. B. Wu, R. Chen, and K. Xia, "Dynamic tensile failure of rocks under static pre-tension," International Journal of Rock Mechanics and Mining Sciences, vol. 80, pp. 12-18, 2015.

[9] S. Carmona and A. Aguado, "New model for the indirect determination of the tensile stress-strain curve of concrete by means of the Brazilian test," Materials and Structures, vol. 45, no. 10, pp. 1473-1485, 2012.

[10] J. Zhao and H. B. Li, "Experimental determination of dynamic tensile properties of a granite," International Journal of Rock Mechanics and Mining Sciences, vol. 37, no. 5, pp. 861-866, 2000.

[11] Q. Z. Wang, W. Li, and H. P. Xie, "Dynamic split tensile test of flattened Brazilian disc of rock with SHPB setup," Mechanics of Materials, vol. 41, no. 3, pp. 252-260, 2009. 
[12] S. Huang, K. Xia, F. Yan, and X. Feng, "An experimental study of the rate dependence of tensile strength softening of longyou sandstone," Rock Mechanics and Rock Engineering, vol. 43, no. 6, pp. 677-683, 2010.

[13] J. Zhao, H. B. Li, and Y. H. Zhao, "Dynamics strength tests of the Bukit Timah granite," Geotechnical Research Report NTU/ GT/98-2, Nanyang Technological University, Singapore, 1998.

[14] S. P. Howe, W. Goldsmith, and J. L. Sackman, "Macroscopic static and dynamic mechanical properties of Yule marblethe mechanical properties of a commercially purchased slab of Yule marble are investigated primarily as a function of strain rate, without consideration of the effects of other parameters," Experimental Mechanics, vol. 14, no. 9, pp. 337-346, 1974.

[15] M. Cai, P. K. Kaiser, F. Suorineni, and K. Su, "A study on the dynamic behavior of the Meuse/Haute-Marne argillite," Physics and Chemistry of the Earth, vol. 32, no. 8-14, pp. 907-916, 2007.

[16] K. Xia, M. H. B. Nasseri, B. Mohanty, F. Lu, R. Chen, and S. N. Luo, "Effects of microstructures on dynamic compression of Barre granite," International Journal of Rock Mechanics and Mining Sciences, vol. 45, no. 6, pp. 879-887, 2008.

[17] W. Goldsmith, J. L. Sackman, and C. Ewerts, "Static and dynamic fracture strength of Barre granite," International Journal of Rock Mechanics and Mining Sciences and, vol. 13, no. 11, pp. 303309, 1976.

[18] F. Dai, S. Huang, K. Xia, and Z. Tan, "Some fundamental issues in dynamic compression and tension tests of rocks using split Hopkinson pressure bar," Rock Mechanics and Rock Engineering, vol. 43, no. 6, pp. 657-666, 2010.

[19] C. R. Siviour, M. R. Arthington, E. Wielewski, and N. Petrinic, "Increasing data from high rate characterization experiments using optical reconstruction," in Proceedings of the AIP Conference, vol. 1426, no. 1, pp. 438-441, 2012.

[20] Z. X. Zhang, S. Q. Kou, L. G. Jiang, and P.-A. Lindqvist, "Effects of loading rate on rock fracture: fracture characteristics and energy partitioning," International Journal of Rock Mechanics and Mining Sciences, vol. 37, no. 5, pp. 745-762, 2000.

[21] Q. B. Zhang and J. Zhao, "A review of dynamic experimental techniques and mechanical behaviour of rock materials," Rock Mechanics and Rock Engineering, vol. 47, no. 4, pp. 1411-1478, 2014.

[22] Y. Mahmutoğlu, "The effects of strain rate and saturation on a micro-cracked marble," Engineering Geology, vol. 82, no. 3, pp. 137-144, 2006.

[23] R. H. C. Wong, K. T. Chau, and P. Wang, "Microcracking and grain size effect in Yuen Long marbles," International Journal of Rock Mechanics and Mining Sciences and Geomechanics, vol. 33, no. 5, pp. 479-485, 1996.

[24] Y. H. Hatzor and V. Palchik, "The influence of grain size and porosity on crack initiation stress and critical flaw length in dolomites," International Journal of Rock Mechanics and Mining Sciences, vol. 34, no. 5, pp. 805-816, 1997.

[25] W. F. Brace, "Dependence of fracture strength of rocks on grain size , Bulletin of the Mineral Industries Experiment Station," Bulletin of the Mineral Industries Experiment Station, Mining Engineering Series. Rock Mechanic, vol. 76, pp. 99-103, 1961.

[26] P. L. P. Wasantha, P. G. Ranjith, J. Zhao, S. S. Shao, and G. Permata, "Strain rate effect on the mechanical behaviour of sandstones with different grain sizes," Rock Mechanics and Rock Engineering, vol. 48, no. 5, pp. 1883-1895, 2014.

[27] E. Eberhardt, B. Stimpson, and D. Stead, "Effects of grain size on the initiation and propagation thresholds of stress-induced brittle fractures," Rock Mechanics and Rock Engineering, vol. 32, no. 2, pp. 81-99, 1999.

[28] W. C. Zhu, L. L. Niu, S. H. Li, and Z. H. Xu, "Dynamic Brazilian test of rock under intermediate strain rate: pendulum hammerdriven shpb test and numerical simulation," Rock Mechanics and Rock Engineering, vol. 48, no. 5, pp. 1867-1881, 2014.

[29] W. Yao, Y. Xu, W. Wang, and P. Kanopolous, "Dependence of dynamic tensile strength of longyou sandstone on heat-treatment temperature and loading rate," Rock Mechanics and Rock Engineering, vol. 49, no. 10, pp. 3899-3915, 2016.

[30] Z. Y. Liao, J. B. Zhu, K. W. Xia, and C. A. Tang, "Determination of dynamic compressive and tensile behavior of rocks from numerical tests of split hopkinson pressure and tension bars," Rock Mechanics and Rock Engineering, vol. 49, no. 10, pp. 39173934, 2016.

[31] S. H. Cho, Y. J. Ogata, and K. Kaneko, "Strain-rate dependency of the dynamic tensile strength of rock," International Journal of Rock Mechanics and Mining Sciences, vol. 40, no. 5, pp. 763-777, 2003.

[32] L. B. Freund, Dynamic Fracture Mechanics, Cambridge University Press, Cambridge, UK, 1990.

[33] C. H. Johansson and R. A. Person, Detonics of High Explosives, Academic Press, New York, NY, USA, 1970.

[34] Q. Niu and D. Xiong, "The research on wave impedance of explosives and rocks," Nonferrous Metals, vol. 40, no. 4, pp. 1317, 1989.

[35] R. B. Clay, M. A. Cook, and R. T. Keyes, "Shock waves in solids and rock mechanics," Mining Research, vol. 2, pp. 681-712, 1962.

[36] P. K. Kaiser and M. Cai, "Design of rock support system under rockburst condition," Journal of Rock Mechanics and Geotechnical Engineering, vol. 4, no. 3, pp. 215-227, 2012.

[37] X. Yi and P. K. Kaiser, "Impact testing for rockbolt design in rockburst conditions," International Journal of Rock Mechanics and Mining Sciences and, vol. 31, no. 6, pp. 671-685, 1994. 


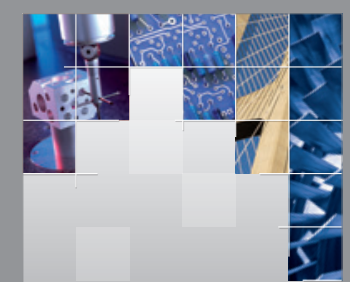

\section{Enfincering}
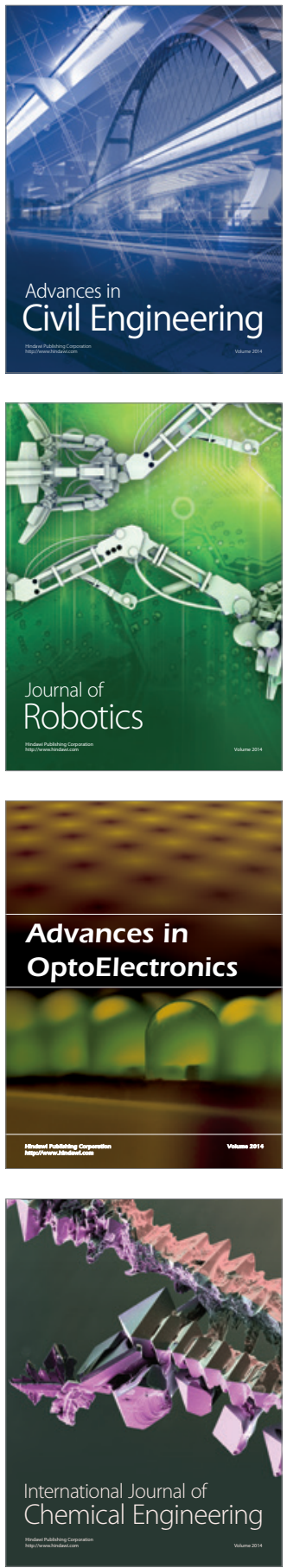

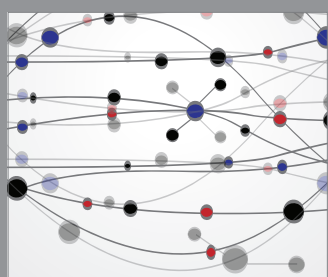

The Scientific World Journal

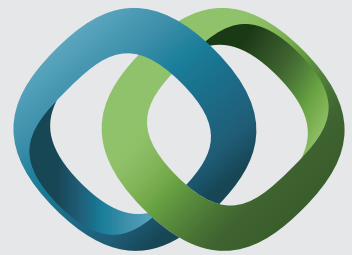

\section{Hindawi}

Submit your manuscripts at

https://www.hindawi.com
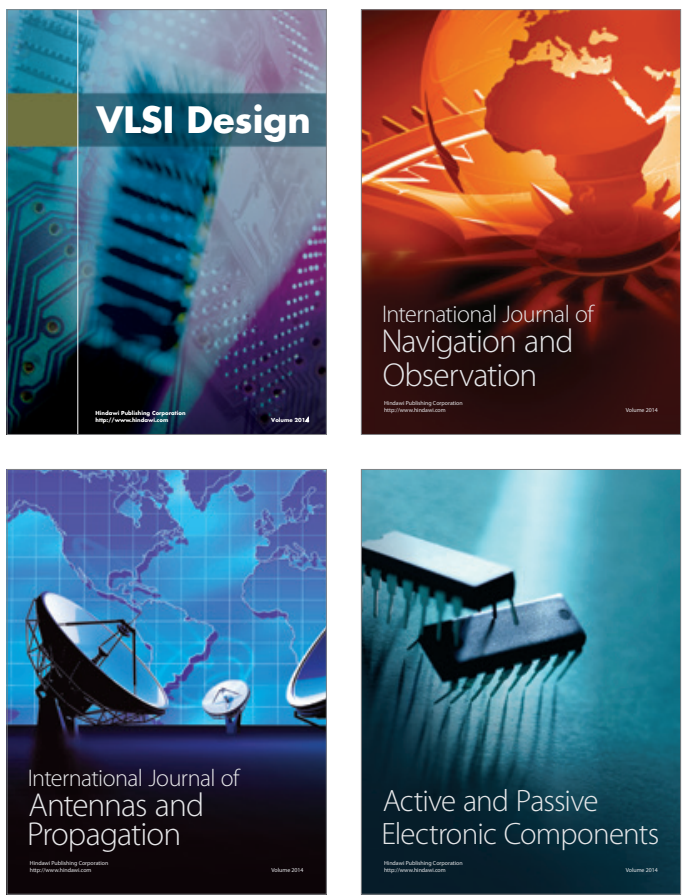
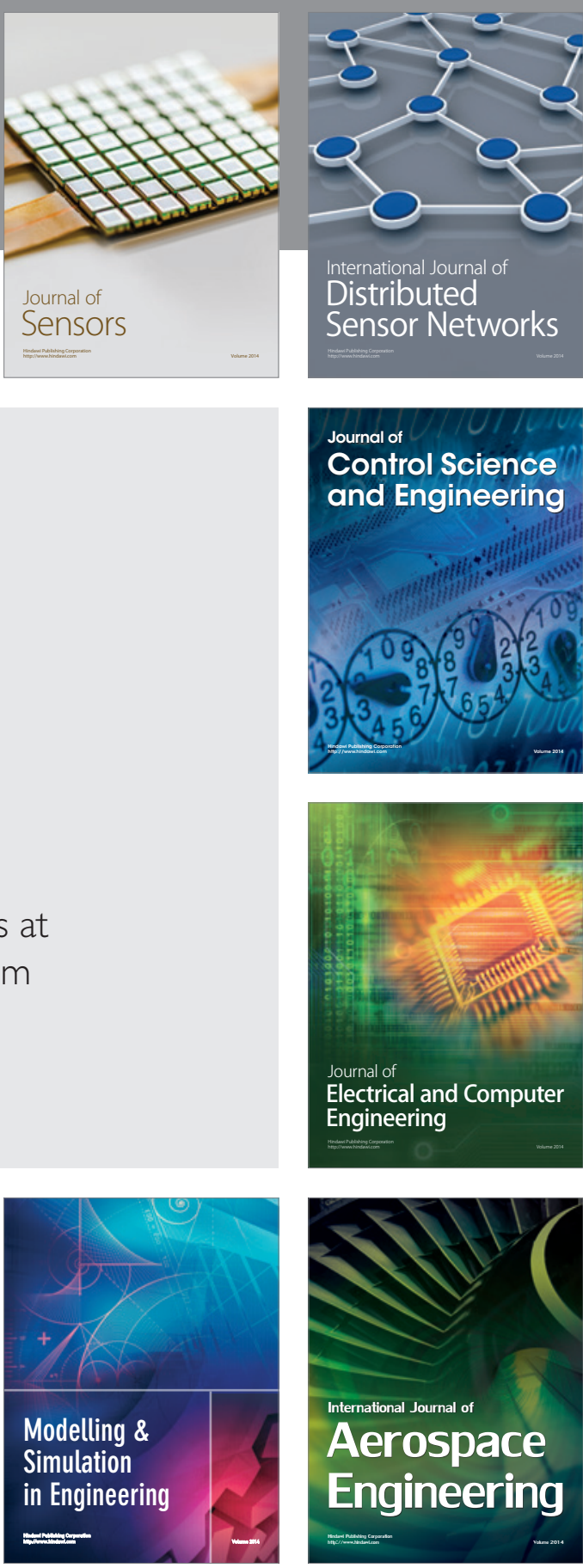

International Journal of

Distributed

Sensor Networks

$-$

Joumal of

Control Science

and Engineering
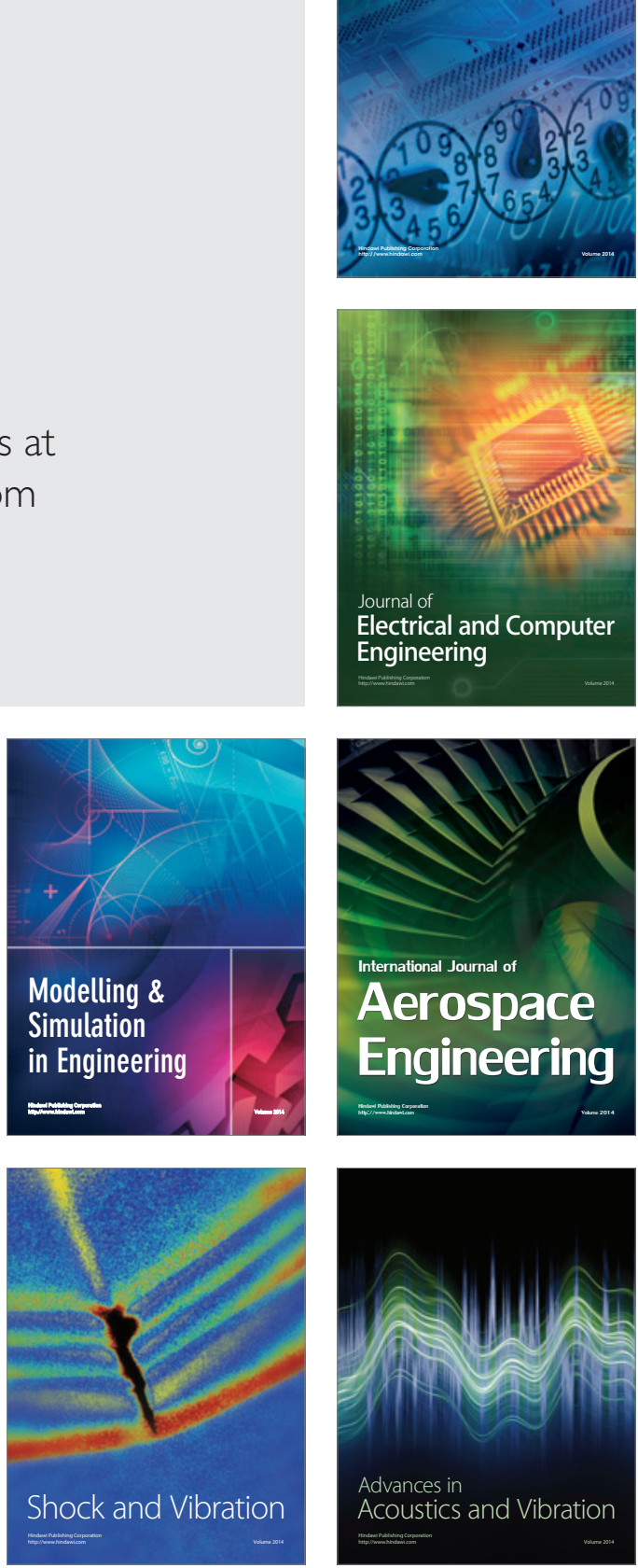